\title{
HEEGAARD DIAGRAMS AND FLOER HOMOLOGY
}

\author{
PETER OZSVÁTH AND ZOLTÁN SZABÓ
}

\begin{abstract}
We review the construction of Heegaard Floer homology for closed threemanifolds and also for knots and links in the three-sphere. We also discuss three applications of this invariant to knot theory: studying the Thurston norm of a link complement, the slice genus of a knot, and the unknotting number of a knot. We emphasize the application to the Thurston norm, and illustrate the theory in the case of the Conway link.
\end{abstract}

\section{HeegaArd Floer homology of three-manifolds}

Floer homology was initially introduced by Floer to study questions in Hamiltonian dynamics [8]. The basic set-up for his theory involves a symplectic manifold $(M, \omega)$, and a pair of Lagrangian submanifolds $L_{0}$ and $L_{1}$. His invariant, Lagrangian Floer homology, is the homology group of a chain complex generated freely by intersection points between $L_{0}$ and $L_{1}$, endowed with a differential which counts pseudo-holomorphic disks. This chain complex arises from a suitable interpretation of the Morse complex in a certain infinite-dimensional setting.

Soon after formulating Lagrangian Floer homology, Floer realized that his basic principles could also be used to construct a three-manifold invariant, instanton Floer homology, closely related to Donaldson's invariants for four-manifolds. In this version, the basic set-up involves a closed, oriented three-manifold $Y$ (satisfying suitable other topological restrictions on $Y$; for example, the theory is defined when $Y$ has trivial integral first homology). Again, one forms a chain complex, but this time the generators are $S U(2)$ representations of the fundamental group of $Y$ (or some suitable perturbation thereof), and the differentials count anti-self-dual Yang-Mills connections on the product of $Y$ with the real line. This invariant plays a crucial role in Donaldson's invariants for smooth four-manifolds: for a four-manifold-with-boundary, the relative Donaldson invariant is a homology class in the instanton Floer homology groups of its boundary [4].

In the present note, we will outline an adaptation of Lagrangian Floer homology, Heegaard Floer homology, which gives rise to a closed three-manifold invariant [33, 32. This invariant also fits into a four-dimensional framework [27. There is a related

Key words and phrases. Heegaard diagrams, Floer homology, Thurston norm.

PSO was supported by NSF grant number DMS-0505811.

ZSz was supported by NSF grant number DMS-0406155. 
invariant of smooth four-manifolds, and indeed relative invariants for this four-manifold invariant take values in the Heegaard Floer homology groups of its boundary.

A Heegaard diagram is a triple consisting of a closed, oriented two-manifold $\Sigma$ of genus $g$, and a pair of $g$-tuples of embedded, disjoint, homologically linearly independent curves $\boldsymbol{\alpha}=\left\{\alpha_{1}, \ldots, \alpha_{g}\right\}$ and $\boldsymbol{\beta}=\left\{\beta_{1}, \ldots, \beta_{g}\right\}$. A Heegaard diagram uniquely specifies a three-manifold, obtained as a union of two genus $g$ handlebodies $U_{\alpha}$ and $U_{\beta}$. In $U_{\alpha}$, the curves $\alpha_{i}$ bound disks, while in $U_{\beta}$, the curves $\beta_{i}$ bound disks. We associate to this data a suitable version of Lagrangian Floer homology.

Our ambient manifold in this case is the $g$-fold symmetric product of $\Sigma$, the set of unordered $g$-tuples of points in $\Sigma$. This space inherits a natural complex structure from a complex structure over $\Sigma$. Inside this manifold, there is a pair of $g$-dimensional real tori, $\mathbb{T}_{\alpha}=\alpha_{1} \times \ldots \times \alpha_{g}$ and $\mathbb{T}_{\beta}=\beta_{1} \times \ldots \times \beta_{g}$. We fix also a reference point

$$
w \in \Sigma-\alpha_{1}-\ldots-\alpha_{g}-\beta_{1}-\ldots-\beta_{g} .
$$

This gives rise to a subvariety $V_{w}=\{w\} \times \operatorname{Sym}^{g-1}(\Sigma) \subset \operatorname{Sym}^{g}(\Sigma)$. We consider the chain complex generated by intersection points $\mathbb{T}_{\alpha} \cap \mathbb{T}_{\beta}$. Concretely, an intersection point of $\mathbb{T}_{\alpha}$ and $\mathbb{T}_{\beta}$ corresponds to a permutation $\sigma$ in the symmetric group on $g$ letters, together with a $g$-tuple of points $\mathbf{x}=\left(x_{1}, \ldots, x_{g}\right)$ with $x_{i} \in \alpha_{i} \cap \beta_{\sigma(i)}$.

The differential again counts holomorphic disks; but some aspect of the homotopy class of the disk is recorded. We make this precise presently. For fixed $\mathbf{x}, \mathbf{y} \in \mathbb{T}_{\alpha} \cap \mathbb{T}_{\beta}$, let $\pi_{2}(\mathbf{x}, \mathbf{y})$ denote the space of homotopy classes of Whitney disks connecting $\mathbf{x}$ to $\mathbf{y}$, i.e. continuous maps of the unit disk $\mathbb{D} \subset \mathbb{C}$ into $\operatorname{Sym}^{g}(\Sigma)$, mapping the part of the boundary of $\mathbb{D}$ with negative resp. positive real part to $\mathbb{T}_{\alpha}$ resp. $\mathbb{T}_{\beta}$, and mapping $i$ resp. $-i$ to $\mathbf{x}$ resp. $\mathbf{y}$. The algebraic intersection number of $\phi \in \pi_{2}(\mathbf{x}, \mathbf{y})$ with the subvariety $V_{w}$ determines a well-defined map

$$
n_{w}: \pi_{2}(\mathbf{x}, \mathbf{y}) \longrightarrow \mathbb{Z} .
$$

It is also useful to think of the two-chain $\mathcal{D}(\phi)$, which is gotten as a formal sum of regions in $\Sigma-\alpha_{1}-\ldots-\alpha_{g}-\beta_{1}-\ldots-\beta_{g}$, where a region is counted with multiplicity $n_{p}(\phi)$, where here $p \in \Sigma$ is any point in this region. Given a Whitney disk, we can consider its space of holomorphic representatives $\mathcal{M}(\phi)$, using the induced complex structure on $\operatorname{Sym}^{g}(\Sigma)$. If this space is non-empty for all choices of almost-complex structure, then the associated two-chain $\mathcal{D}(\phi)$ has only non-negative local multiplicities. The group $\mathbb{R}$ acts on $\mathcal{M}(\phi)$ by translation. The moduli space $\mathcal{M}(\phi)$ has an expected dimension $\mu(\phi)$, which is obtained as the Fredholm index of the linearized $\bar{\partial}$-operator. This quantity, the Maslov index, is denoted $\mu(\phi)$.

It is sometimes necessary to perturb the holomorphic condition to guarantee that moduli spaces are manifolds of the expected dimension. It is useful (though slightly imprecise) to think of a holomorphic disk in $\mathcal{M}(\phi)$ as a pair consisting of a holomorphic surface $F$ with marked boundary, together with a degree $g$ holomorphic projection map $\pi$ from $F$ to the standard disk, and also a map $f$ from $F$ into $\Sigma$. Here, $f$ maps $\pi^{-1}$ of 
the subarc of the boundary of $\mathbb{D}$ with negative resp. positive real part into the subset $\alpha_{1} \cup \ldots \cup \alpha_{g} \operatorname{resp} \beta_{1} \cup \ldots \cup \beta_{g}$.

We now consider the complex $C F^{-}(Y)$ which is the free $\mathbb{Z}[U]$-module generated by $\mathbb{T}_{\alpha} \cap \mathbb{T}_{\beta}$, with differential given by

$$
\partial \mathbf{x}=\sum_{\mathbf{y} \in \mathbb{T}_{\alpha} \cap \mathbb{T}_{\beta}} \sum_{\left\{\phi \in \pi_{2}(\mathbf{x}, \mathbf{y}) \mid \mu(\phi)=1\right\}} \#\left(\frac{\mathcal{M}(\phi)}{\mathbb{R}}\right) U^{n_{w}(\phi)} \mathbf{y}
$$

In the case where $Y$ is an integral homology sphere, the above sum is readily seen to be finite. (In the case where the first Betti number is positive, some further constraints must be placed on the Heegaard diagram.) With the help of Gromov's compactification of the space pseudo-holomorphic curves [14, one can see that $\partial^{2}=0$.

According to 33, the homology groups $H^{-}(Y)$ of $C F^{-}(Y)$ are a topological invariant of $Y$. Indeed, the chain homotopy type of $C F^{-}(Y)$ is a topological invariant, and, since $C F^{-}(Y)$ is a module over $\mathbb{Z}[U]$, there are a number of other associated constructions. For example, we can form the chain complex $C F^{\infty}(Y)$ obtained by inverting $U$, i.e. a chain complex over $\mathbb{Z}\left[U, U^{-1}\right]$, with differential as in Equation (11). The quotient of $C F^{\infty}(Y)$ by $C F^{-}(Y)$ is a complex $C F^{+}(Y)$ which is often more convenient to work with. The corresponding homology groups are denoted $H F^{\infty}(Y)$ and $H F^{+}(Y)$ respectively. Also, there is a chain complex $\widehat{C F}$ obtained by setting $U=0$; explicitly, it is generated freely over $\mathbb{Z}$ by $\mathbb{T}_{\alpha} \cap \mathbb{T}_{\beta}$, and endowed with the differential

$$
\widehat{\partial} \mathbf{x}=\sum_{\mathbf{y} \in \mathbb{T}_{\alpha} \cap \mathbb{T}_{\beta}} \sum_{\left\{\phi \in \pi_{2}(\mathbf{x}, \mathbf{y}) \mid\right.} \#\left(\frac{\mathcal{M}(\phi)}{\mathbb{R}}\right) \mathbf{y}
$$

and its homology (also a topological invariant of $Y$ ) is denoted $\widehat{H F}(Y)$.

The invariants $H F^{-}(Y), H F^{\infty}(Y)$, and $H F^{+}(Y)$, together with the exact sequence connecting them, are crucial ingredients in the construction of a Heegaard Floer invariant $\Phi$ for closed, smooth four-manifolds. We will say only little more about this invariant here, referring the reader to [27] for its construction.

\section{Heegaard Floer homology of knots}

Heegaard Floer homology for three-manifolds has a refinement to an invariant for null-homologous knots in a three-manifold, as defined in [31, and also independently by Rasmussen in 38.

A knot $K$ in a three-manifold $Y$ is specified by a Heegaard diagram $(\Sigma, \boldsymbol{\alpha}, \boldsymbol{\beta})$ for $Y$, together with a pair $w$ and $z$ of basepoints in $\Sigma$. The knot $K$ is given as follows. Connect $w$ and $z$ by an arc $\xi$ in $\Sigma-\alpha_{1}-\ldots-\alpha_{g}$ and an arc $\eta$ in $\Sigma-\beta_{1}-\ldots-\beta_{g}$. The $\operatorname{arcs} \xi$ and $\eta$ are then pushed into $U_{\alpha}$ and $U_{\beta}$ respectively, so that they both meet $\Sigma$ only at $w$ and $z$, giving new $\operatorname{arcs} \xi^{\prime}$ and $\eta^{\prime}$. Our knot $K$, then, is given by $\xi^{\prime}-\eta^{\prime}$. For 
simplicity, we consider here the case where the ambient manifold $Y$ is the three-sphere $S^{3}$.

The new basepoint $z$ gives the Heegaard Floer complex a filtration. Specifically, we can construct a map

$$
F: \mathbb{T}_{\alpha} \cap \mathbb{T}_{\beta} \longrightarrow \mathbb{Z}
$$

by

$$
F(\mathbf{x})-F(\mathbf{y})=n_{z}(\phi)-n_{w}(\phi)
$$

where $\phi \in \pi_{2}(\mathbf{x}, \mathbf{y})$. It is easy to see that this quantity is independent of the choice of $\phi$, depending only on $\mathbf{x}$ and $\mathbf{y}$. Moreover, if $\mathbf{y}$ appears with non-zero multiplicity in $\widehat{\partial}(\mathbf{x})$, then $F(\mathbf{x}) \geq F(\mathbf{y})$. This follows from the fact that there is a pseudo-holomorphic disks $\phi \in \pi_{2}(\mathbf{x}, \mathbf{y})$ with $n_{w}(\phi)=0$, and also $n_{z}(\phi) \geq 0$, since a pseudo-holomorphic disks meets the subvariety $V_{z}$ with non-negative intersection number.

Equation (2) defines $F$ uniquely up to an overall translation. This indeterminacy will be removed presently.

The filtered chain homotopy type of this filtered chain complex is an invariant of the knot $K$. For example, the homology of the associated graded object, the knot Floer homology is an invariant of $K \subset S^{3}$, defined by

$$
\widehat{\operatorname{HFK}}\left(S^{3}, K\right)=\bigoplus_{s \in \mathbb{Z}} \widehat{\operatorname{HFK}}\left(S^{3}, K, s\right),
$$

where $\widehat{\operatorname{HFK}}\left(S^{3}, K, s\right)$ is the homology group of the chain complex generated by intersection points $\mathbf{x} \in \mathbb{T}_{\alpha} \cap \mathbb{T}_{\beta}$ with $F(\mathbf{x})=s$, endowed with differential

$$
\partial \mathbf{x}=\sum_{\mathbf{y} \in \mathbb{T}_{\alpha} \cap \mathbb{T}_{\beta}} \sum_{\left\{\begin{array}{c}
\mu(\phi)=1, \\
\left\{\in \pi_{2}(\mathbf{x}, \mathbf{y}) \mid\right. \\
n_{w}(\phi)=n_{z}(\phi)=0
\end{array}\right\}} \#\left(\frac{\mathcal{M}(\phi)}{\mathbb{R}}\right) \mathbf{y}
$$

The graded Euler characteristic of this theory is the Alexander polynomial of $K$, in the sense that

$$
\Delta_{K}(T)=\sum_{s \in \mathbb{Z}} \chi\left(\widehat{\operatorname{HFK}}_{*}(K, s)\right) \cdot T^{s}
$$

This formula can be used to pin down the additive indeterminacy of $F$ : we require that $F$ be chosen so that the graded Euler characteristic is the symmetrized Alexander polynomial. In fact, this symmetry has a stronger formulation, as a relatively graded isomorphism

$$
\widehat{\operatorname{HFK}}_{*}(K, s) \cong \widehat{\operatorname{HFK}}_{*}(K,-s)
$$




\section{HeegaArd Floer homology for links}

Heegaard Floer homology groups of knots can be generalized to the case of links in $S^{3}$. For an $\ell$-component link, we consider a Heegaard diagram with genus $g$ Heegaard surface, and two $(g+\ell-1)$-tuples attaching circles $\boldsymbol{\alpha}=\left\{\alpha_{1}, \ldots, \alpha_{g+\ell-1}\right\}$ and $\boldsymbol{\beta}=\left\{\beta_{1}, \ldots, \beta_{g+\ell-1}\right\}$. We require $\left\{\alpha_{1}, \ldots, \alpha_{g+\ell-1}\right\}$ to be disjoint and embedded, and to span a $g$-dimensional lattice in $H_{1}(\Sigma ; \mathbb{Z})$. The same is required of the $\left\{\beta_{1}, \ldots, \beta_{g+\ell-1}\right\}$. Clearly, $\Sigma-\alpha_{1}-\ldots-\alpha_{g+\ell-1}$ consists of $\ell$ components $A_{1}, \ldots, A_{\ell}$. Similarly, $\Sigma-\beta_{1}-$ $\ldots-\beta_{g+\ell-1}$ consists of $\ell$ components $B_{1}, \ldots, B_{\ell}$. We assume that this Heegaard diagram has the special property that $A_{i} \cap B_{i}$ is non-empty. Indeed, for each $i=1, \ldots, \ell$, we choose basepoints $w_{i}$ and $z_{i}$ to lie inside $A_{i} \cap B_{i}$. We call the collection of data $\left(\Sigma, \boldsymbol{\alpha}, \boldsymbol{\beta},\left\{w_{1}, \ldots, w_{\ell}\right\},\left\{z_{1}, \ldots, z_{\ell}\right\}\right)$ a $2 \ell$-pointed Heegaard diagram.

A link can now be constructed in the following manner. Connect $w_{i}$ and $z_{i}$ by an arc $\xi_{i}$ in $A_{i}$ and an arc $\eta_{i}$ in $B_{i}$. Again, the arc $\xi_{i}$ resp. $\eta_{i}$ is pushed into $U_{\alpha}$ resp. $U_{\beta}$ to give rise to a pair of $\operatorname{arcs} \xi_{i}^{\prime}$ and $\eta_{i}^{\prime}$. The link $L$ is given by $\cup_{i=1}^{\ell} \xi_{i}^{\prime}-\eta_{i}^{\prime}$. For a $2 \ell$-pointed Heegaard diagram for $S^{3}\left(\Sigma, \boldsymbol{\alpha}, \boldsymbol{\beta},\left\{w_{1}, \ldots, w_{\ell}\right\},\left\{z_{1}, \ldots z_{\ell}\right\}\right)$, if $L$ is the link obtained in this manner, we say that the Heegaard diagram is compatible with the link $L$.

We will need to make an additional assumption on the Heegaard diagram. A periodic domain is a two-chain in $\Sigma$ of the form

$$
\sum c_{i}\left(A_{i}-B_{i}\right)
$$

where $c_{i} \in \mathbb{Z}$. Our assumption is that all non-zero periodic domains have some positive and some negative local multiplicities $c_{i}$. This assumption on the pointed Heegaard diagram is called admissibility.

Let $L \subset S^{3}$ be an $\ell$-component link, suppose that $L$ is embedded so that the restriction of the height function to $L$ has $b$ local maxima, then we can construct a compatible $2 \ell$-pointed heegaard diagram with Heegaard genus $g=b-\ell$.

For example, consider the two-component "Conway link" pictured in Figure 11. This is the $(2,-3,-2,3)$ pretzel link, also known as L10n59 in Thistlethwaite's link table [1].

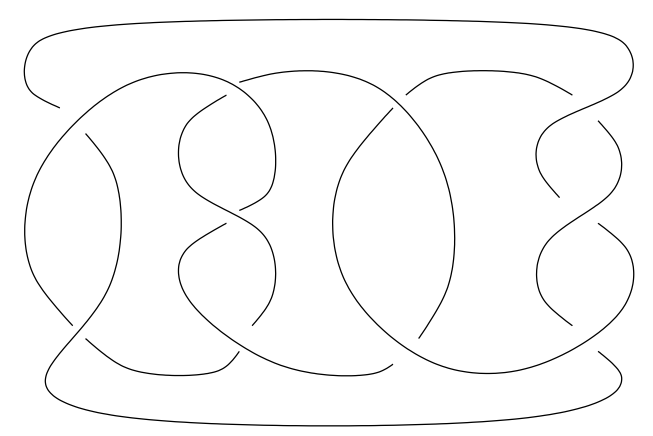

Figure 1. The Conway link. 
For this link, $b=4$, and hence we can draw it on a surface of genus $g=2$, as illustrated in Figure 2. It is straightforward to verify that the space of periodic domains

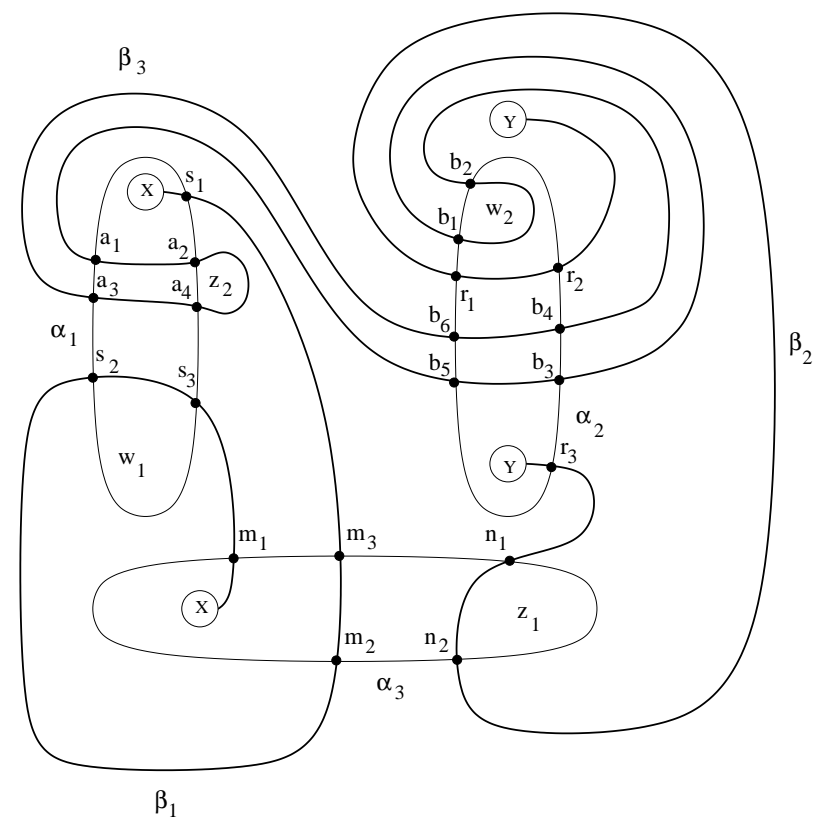

Figure 2. Pointed Heegaard diagram for the Conway link. This picture takes place on the genus two surface obtained by identifying the two disks labeled by $X$ and the two disks labeled by $Y$.

is one-dimensional; drawing a picture of this generator, it is also straightforward to see that the diagram is admissible.

Now, we work inside $\operatorname{Sym}^{g+\ell-1}(\Sigma)$, relative to the tori $\mathbb{T}_{\alpha}=\alpha_{1} \times \ldots \times \alpha_{g+\ell-1}$ and $\mathbb{T}_{\beta}=\beta_{1} \times \ldots \times \beta_{g+\ell-1}$, and consider intersection points of $\mathbb{T}_{\alpha}$ and $\mathbb{T}_{\beta}$; i.e. $g+\ell-1$-tuples of points $\left(x_{1}, \ldots, x_{g+\ell-1}\right)$ with $x_{i} \in \alpha_{i} \cap \beta_{\sigma(i)}$ for some permutation $\sigma$ in the symmetric group on $g+\ell-1$ letters. We then form the chain complex $\widehat{\mathrm{CF} L}\left(S^{3}, L\right)$ generated freely by these intersection points.

For example, for the figure illustrated in Figure 2, the curves $\alpha_{i}$ and $\beta_{j}$ intersect according to the pattern

\begin{tabular}{c|ccc}
$\cap$ & $\alpha_{1}$ & $\alpha_{2}$ & $\alpha_{3}$ \\
\hline$\beta_{1}$ & $\left\{s_{1}, s_{2}, s_{3}\right\}$ & $\emptyset$ & $\left\{m_{1}, m_{2}, m_{3}\right\}$ \\
$\beta_{2}$ & $\emptyset$ & $\left\{r_{1}, r_{2}, r_{3}\right\}$ & $\left\{n_{1}, n_{2}\right\}$ \\
$\beta_{3}$ & $\left\{a_{1}, \ldots, a_{4}\right\}$ & $\left\{b_{1}, \ldots, b_{6}\right\}$ & $\emptyset$
\end{tabular}

Now, there are exactly two permutations of $\{1,2,3\}$ for which $\alpha_{i} \cap \beta_{\sigma(i)}$ is non-trivial for all $i$. This gives two types of intersection points of $\mathbb{T}_{\alpha} \cap \mathbb{T}_{\beta}$, namely, $a_{i} \times m_{j} \times r_{k}$ 
(with $i=1, \ldots, 4, j=1, \ldots, 3, k=1, \ldots, 3)$ and also $b_{i} \times n_{j} \times s_{k}($ with $i=1, \ldots, 6, j=1,2$, $k=1,2,3)$. This gives a chain complex with a total of 72 generators.

The complex $\widehat{\mathrm{CFL}}$ has a grading, the Maslov grading, which is specified up to overall translation by the convention

$$
\operatorname{gr}(\mathbf{x})-\operatorname{gr}(\mathbf{y})=\mu(\phi)-2 \sum_{i=1}^{\ell} n_{w_{i}}(\phi),
$$

where $\phi \in \pi_{2}(\mathbf{x}, \mathbf{y})$ is any Whitney disk connecting $\mathbf{x}$ and $\mathbf{y}$. The parity of the Maslov grading depends on the local sign of the intersection number of $\mathbb{T}_{\alpha}$ and $\mathbb{T}_{\beta}$ at $\mathbf{x}$.

But $\widehat{\mathrm{CFL}}$ has an additional grading, the $\mathbb{H}$-grading. To define this, we associate to each $\phi \in \pi_{2}(\mathbf{x}, \mathbf{y})$ the pair of vectors

$$
n_{\mathbf{w}}(\phi)=\left(n_{w_{1}}(\phi), \ldots, n_{w_{\ell}}(\phi)\right) \text { and } n_{\mathbf{z}}(\phi)=\left(n_{z_{1}}(\phi), \ldots, n_{z_{\ell}}(\phi)\right) .
$$

We have a function $F: \mathbb{T}_{\alpha} \cap \mathbb{T}_{\beta} \longrightarrow \mathbb{Z}^{\ell} \cong H_{1}\left(S^{3}-L ; \mathbb{Z}\right.$ ) (where the latter identification is given by the meridians of the link $L$ ) specified uniquely up to translation by the formula

$$
F(\mathbf{x})-F(\mathbf{y})=n_{\mathbf{z}}(\phi)-n_{\mathbf{w}}(\phi),
$$

where $\phi$ is any choice of homotopy class in $\pi_{2}(\mathbf{x}, \mathbf{y})$.

Endow $\widehat{\mathrm{CFL}}\left(S^{3}, L\right)$ with the differential

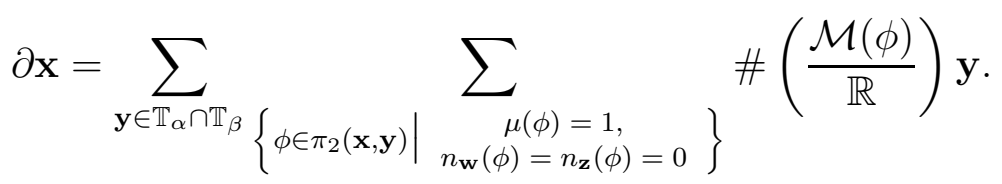

It is easy to see that this differential drops Maslov grading by one. Moreover, the complex naturally splits into summands indexed by elements of $\mathbb{Z}^{\ell} \cong H_{1}\left(S^{3}-L ; \mathbb{Z}\right)$ specified by the function $F$. We find it natural to think of these summands, in fact, as indexed by the affine lattice $\mathbb{H} \subset H_{1}\left(S^{3}-L ; \mathbb{R}\right)$ over $H_{1}\left(S^{3}-L ; \mathbb{Z}\right)$, given by elements

$$
\sum_{i=1}^{\ell} a_{i} \cdot\left[\mu_{i}\right]
$$

where $a_{i} \in \mathbb{Q}$ satisfies the property that

$$
2 a_{i}+\operatorname{lk}\left(L_{i}, L-L_{i}\right)
$$

is an even integer. The translational ambiguity of the map is then pinned down by the following generalization of Equation (4):

$$
\widehat{\mathrm{HFL}}_{*}(\vec{L}, h) \cong \widehat{\mathrm{HFL}}_{*}(\vec{L},-h) .
$$

In practice, it is easy to calculate the difference in $F$ for any two intersection of $\mathbb{T}_{\alpha}$ and $\mathbb{T}_{\beta}$ which have the same type (i.e. same pattern of intersection $\alpha_{i} \cap \beta_{\sigma(i)}$ ). To this end, it suffices to find for each pair of intersection points $x, x^{\prime} \in \alpha_{i} \cap \beta_{j}$, a disk (or 
more generally a compact surface with a single boundary component) in $\Sigma$ which meets $\alpha_{i}$ along one arc in its boundary and $\beta_{j}$ along the complementary arc, carrying the intersection points of the closures of the arcs to $x$ and $x^{\prime}$. We then define the "relative difference" of $x$ and $x^{\prime}, F^{i, j}(x)-F^{i, j}\left(x^{\prime}\right)$, to be $n_{\mathbf{z}}-n_{\mathbf{w}}$ for this disk (or surface). It is easy to see then that if $\mathbf{x}$ and $\mathbf{y} \in \mathbb{T}_{\alpha} \cap \mathbb{T}_{\beta}$ are two intersection points with the same type (as specified by $\sigma$ ), then

$$
F(\mathbf{x})-F(\mathbf{y})=\sum_{i=1}^{g+\ell-1} F^{i, \sigma(i)}\left(x_{i}\right)-F^{i, \sigma(i)}\left(y_{i}\right),
$$

where $\mathbf{x}=\left(x_{1}, \ldots, x_{g+\ell-1}\right)$ and $y=\left(y_{1}, \ldots, y_{g+\ell-1}\right)$. This determines $F(\mathbf{x})-F(\mathbf{y})$ for $\mathbf{x}$ and $\mathbf{y}$ of the same type. Different types can then be compared by choosing homotopy classes connecting them (and in suitable circumstances, the translational ambiguity can be removed using Equation (15) ).

We display the relative differences for the various intersection points for the diagram from Figure 3 .
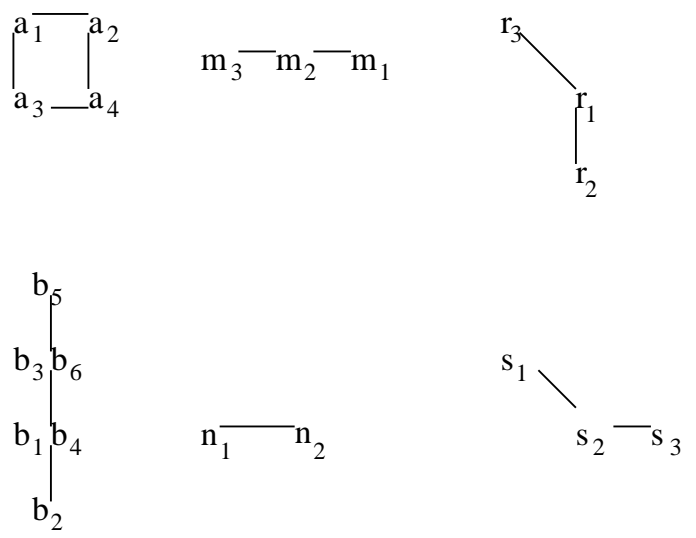

Figure 3. Generators for $\widehat{\mathrm{HFL}}$ of the Conway link. We illustrate the relative differences of the various intersection points of $\alpha_{i}$ and $\beta_{j}$. A horizontal resp. vertical segment denotes two intersection points whose relative difference is one in the first resp. second component; e.g. there is a disk in Figure $2 \phi$ from $a_{2}$ to $a_{1}$ with $n_{\mathbf{z}}-n_{\mathbf{w}}$ given by $(1,0)$, while there is one from $b_{5}$ to $b_{3}$ with relative difference given by $(0,1)$. Finally, for the diagonal edges, we have a disk from $r_{3}$ to $r_{1}$ with relative difference $(-1,1)$.

It is now straightforward to verify that the ranks of the chain groups in each value of $F$ is given as in Figure 4. It is more challenging to calculate the homology groups of $\widehat{\mathrm{CFL}}$. 


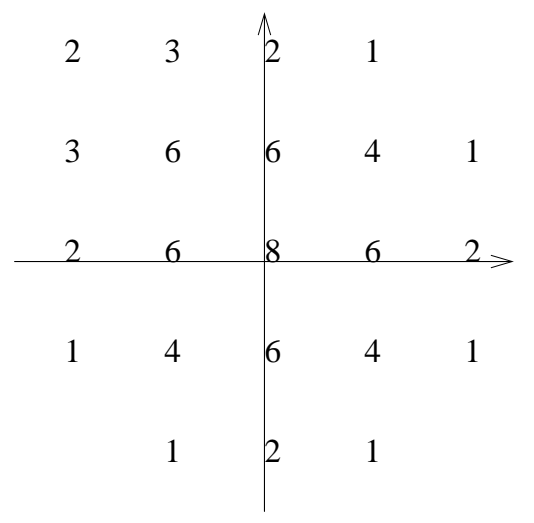

Figure 4. Generators for $\widehat{\mathrm{CF} L}$ of the Conway link. The 72 generators of $\widehat{\mathrm{CFL}}$ coming from the diagram in Figure 2 are separated into different .

Some aspects are immediate. For example, it follows by glancing at Figure 4, and comparing Equation (5) that the homology in $\mathbb{H}$-grading $(-2,2)$ is trivial (as there are no generators in the $\mathbb{H}$-grading $(2,-2))$, and that in $\mathbb{H}$-gradings $(-1,2)$ and $(-2,1)$ the groups $\widehat{\mathrm{HFL}}$ have rank one. This already suffices to determine the convex hull of $h \in \mathbb{H}$ for which $\widehat{\operatorname{HFL}}(L, h)$ is non-trivial, as required for the application to the Thurston norm below (see esp. Equation (17)).

Also, the calculation of $\widehat{\operatorname{HFL}}(L,(x, y))$ with $(x, y) \in\{(0, \pm 2),( \pm 2,0)\}$ follows from the fact that for each of these $\mathbb{H}$-gradings, every generator has the same Maslov grading.

Next, consider the part in $\mathbb{H}$-grading $(1,1)$. There are four generators

$$
\left\{a_{1} \times m_{1} \times r_{1}, \quad a_{2} \times m_{2} \times r_{1}, \quad a_{4} \times r_{3} \times m_{1}, \quad b_{5} \times n_{1} \times s_{3}\right\} .
$$

For the case where $\mathbf{x}=b_{5} \times n_{1} \times s_{3}$ and $\mathbf{y} \in\left\{a_{1} \times m_{1} \times r_{1}, a_{2} \times m_{2} \times r_{1}\right\}$, there is a homotopy class $\phi \in \pi_{2}(\mathbf{x}, \mathbf{y})$ whose associated two-chain $\mathcal{D}(\phi)$ is a hexagon. For the case where $\mathbf{y}=a_{2} \times m_{2} \times r_{1}$, we illustrate this in Figure 5. A hexagon gives rise to a flow-line connecting $\mathbf{x}$ to $\mathbf{y}$. To this end, we think of a holomorphic disk in $\operatorname{Sym}^{3}(\Sigma)$ as a branched triple-cover $F$ of the standard disk, together with a map of $F$ into $\Sigma$. The given hexagonal domain in $\Sigma$ can be realized as a branched triple-cover of the disk $\mathbb{D}$. Moreover, any other domain connecting $\mathbf{x}$ to $\mathbf{y}$ has negative local multiplicity somewhere. Hence, we have that

$$
\partial b_{5} \times n_{1} \times s_{3}=a_{1} \times m_{1} \times r_{1}+a_{2} \times m_{2} \times r_{1} .
$$

It can also be seen that

$$
\operatorname{gr}\left(b_{5} \times n_{1} \times s_{3}\right)=\operatorname{gr}\left(a_{4} \times m_{1} \times r_{3}\right),
$$

but there are no non-negative domains from $a_{4} \times m_{1} \times r_{3}$ to either of $\left\{a_{1} \times m_{1} \times r_{1}, a_{2} \times\right.$ $\left.m_{2} \times r_{1}\right\}$. It follows at once that $\widehat{\operatorname{HFL}}(L,(1,1))$ has rank two. 


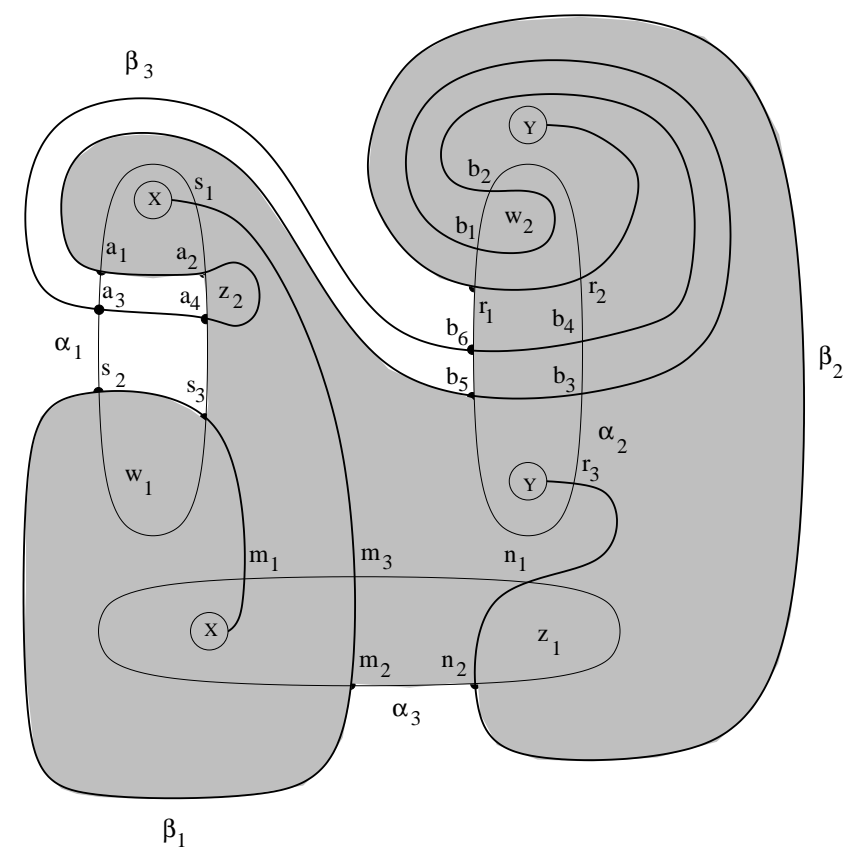

Figure 5. A flowline. The complement of the shaded region gives a hexagon connecting $b_{5} \times n_{1} \times s_{3}$ to $a_{2} \times m_{2} \times r_{1}$.

With some additional work, one can verify that all the link Floer homology groups of the Conway link are as displayed below in Figure 6.

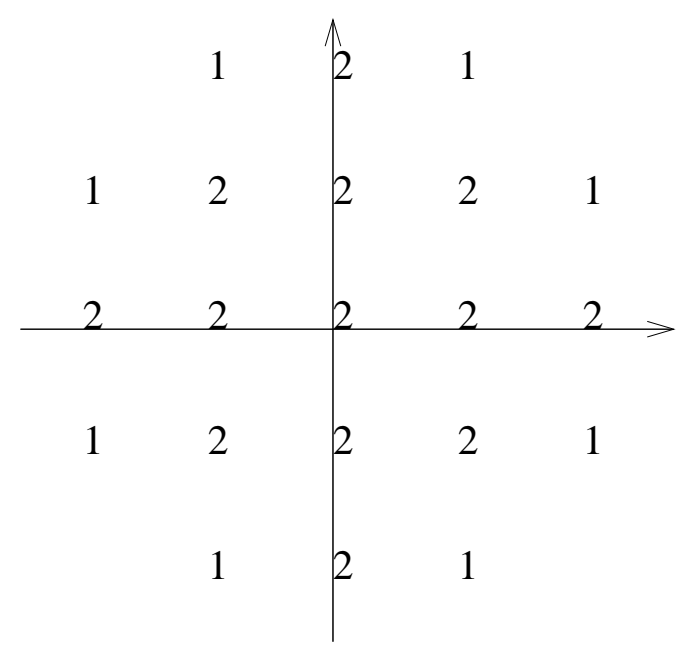

Figure 6. Ranks of $\widehat{\mathrm{HFL}}$ for the Conway link. The ranks are displayed, along with their $\mathbb{H}$ grading, thought of as coordinates in the plane. 


\section{BASIC PROPERTIES}

Perhaps the single most fundamental property of Heegaard Floer homology is that it satisfies an exact triangle for surgeries. More precisely, a triad of three-manifolds $Y_{1}$, $Y_{2}, Y_{3}$ is a cyclically ordered triple of three-manifolds obtained as follows. Let $M$ be a three-manifold with torus boundary, and fix three simple, closed curves in its boundary $\gamma_{1}, \gamma_{2}, \gamma_{3}$ any two of which intersect transversally in one point, and ordered so that there are orientations on the three curves so that

$$
\#\left(\gamma_{1} \cap \gamma_{2}\right)=\#\left(\gamma_{2} \cap \gamma_{3}\right)=\#\left(\gamma_{3} \cap \gamma_{1}\right)=-1 .
$$

We let $Y_{i}$ be the three-manifold obtained by Dehn filling $M$ along the curve $\gamma_{i}$.

Theorem 4.1. Let $Y_{1}, Y_{2}$, and $Y_{3}$ be a triad of three-manifolds. Then, there is an long exact sequence of the form

$$
\ldots \longrightarrow \widehat{H F}\left(Y_{1}\right) \longrightarrow \widehat{H F}\left(Y_{2}\right) \longrightarrow \widehat{H F}\left(Y_{3}\right) \longrightarrow \ldots \text {. }
$$

The maps in the exact triangle are induced by the three natural two-handle cobordisms connecting $Y_{i}$ and $Y_{i+1}$ (where we $i$ as an integer modulo 3), in a manner made precise in [27.

The above surgery exact sequence is similar to an exact sequence established by Floer for instanton Floer homology (only using a restricted class of triads) [9], 2]. An analogous exact sequence has been established for Floer homology of Seiberg-Witten monopoles, see [20]. There are also related exact sequences in symplectic geometry, cf. [40].

Note that there are other variants of the exact triangle, and indeed, there are certain other related calculational techniques for Heegaard Floer homology. For example, for a knot $K$ in an integral homology sphere $Y$, the filtered chain homotopy type of the induced knot invariant can be used to calculate the Floer homology groups of arbitrary surgeries on $K, 28$.

\section{THREE APPLiCATIONS}

Heegaard Floer homology is particularly well suited to problems in knot-theory and three-manifold topology which can be formulated in terms of the existence of fourdimensional cobordisms. We focus here on a few concrete problems which can be formulated for knots and links in the three-sphere. For some other applications, see [36], 34, 22, 37].

5.1. Thurston norm. Let $K \subset S^{3}$ be a knot. The Seifert genus of $K$, denoted $g(K)$, is the minimal genus of any embedded surface $F \subset S^{3}$ with boundary $K$. Clearly, if $g(K)=0$, then $K$ is the unknot. 
According to [30, knot Floer homology detects the Seifert genus of a knot, by the property that

$$
g(K)=\max \{s \mid \widehat{\operatorname{HFK}}(K, s) \neq 0\} .
$$

There is a natural generalization of the knot genus and indeed of Equation (6)). This is best formulated in terms of Thurston's norm on second homology.

Recall that if $F$ is a compact, oriented, but possibly disconnected surface-withboundary $F=\bigcup_{i=1}^{n} F_{i}$, its complexity is given by

$$
\chi_{-}(F)=\sum_{\left\{F_{i} \mid \chi\left(F_{i}\right) \leq 0\right\}}-\chi\left(F_{i}\right) .
$$

Given any homology class $h \in H_{2}\left(S^{3}, L\right)$, it is easy to see that there is a compact, oriented surface-with-boundary embedded in $S^{3}-\operatorname{nd}(K)$ representing $h$. Consider the function from $H^{1}\left(S^{3}-L ; \mathbb{Z}\right)$ to the integers defined by

$$
x(h)=\min _{\left\{F \hookrightarrow S^{3}-\operatorname{nd}(K) \mid[F]=\mathrm{PD}[h]\right\}} \chi_{-}(F) .
$$

Indeed, according to Thurston [41, this function $x$ satisfies an inequality $x\left(h_{1}+h_{2}\right) \leq$ $x\left(h_{1}\right)+x\left(h_{2}\right)$, and it is linear on rays, i.e. given $h \in H_{2}\left(S^{3}, L\right)$ and a non-negative integer $n$, we have that $x(n \cdot h)=n x(h)$. Thus, $x$ can be naturally extended to a seminorm on $H^{1}\left(S^{3}-L ; \mathbb{R}\right)$, the Thurston semi-norm. In fact, this semi-norm is uniquely specified by its unit ball

$$
B_{x}=\left\{h \in H^{1}\left(S^{3}-L ; \mathbb{R}\right) \mid x(h) \leq 1\right\},
$$

which is a polytope $H^{1}\left(S^{3}-L ; \mathbb{R}\right)$ whose vertices lie at lattice points in $H^{1}\left(S^{3}-L ; \mathbb{Z}\right)$.

Equation (6) can now be generalized as follows. A trivial component of a link $L$ is a component $K \subset L$ which is unknotted an geometrically unlinked from the complement $L-K$. Suppose that $L$ is a link with no trivial components. Then, given $s \in H^{1}\left(S^{3}-L\right)$,

$$
x(h)+\sum_{i=1}^{\ell}\left|\left\langle\mu_{i}, h\right\rangle\right|=2 \cdot \max _{\{s \in \mathbb{H} \mid \widehat{\operatorname{HFL}}(L, s) \neq 0\}}\langle h, s\rangle,
$$

where here $\langle$,$\rangle denotes the Kronecker pairing of H_{1}\left(S^{3}-L ; \mathbb{R}\right)$ with $H^{1}\left(S^{3}-L ; \mathbb{R}\right)$.

This formula can be thought of more geometrically from the following point of view. Consider the dual norm $x^{*}: H_{1}\left(S^{3}-L ; \mathbb{R}\right) \longrightarrow \mathbb{R}$ given by

$$
x^{*}(s)=\max _{\left\{h \in B_{x}\right\}}\langle s, h\rangle .
$$

The unit ball $B_{x *}$ is a (possibly degenerate) polytope in $H_{1}\left(S^{3}-L ; \mathbb{R}\right)$ called the dual Thurston polytope. Equation (17) states that for a $\operatorname{link} L$ with no trivial components, if we take the convex hull of the set of $s \in \mathbb{H}$ with $\widehat{\operatorname{HFL}}(L, s)$, and rescale that polytope 
by a factor of two, then we obtain the sum of the dual Thurston polytope with the symmetric hypercube in $H_{1}\left(S^{3}-L ; \mathbb{R}\right)$ with edge-length two.

Of course, the Thurston norm can be defined for closed three-manifolds, as well. In fact, a result analogous to Equation (7) can be proved for closed three-manifolds $Y$, instead of link complements. An analogous result has been shown to hold for SeibergWitten monopole Floer homology [19] (but at present there is no analogue of knot and link Floer homology in gauge-theoretic terms).

Although the statement of Equation (6) does not explicitly involve any four-dimensional theory, the proof of this result does use the full force of Heegaard Floer homology, combined with Gabai's theory of sutured manifolds, and recent results in symplectic geometry. Specifically, according to a combination of theorems of Gabai [11], [12, Eliashberg-Thurston [6, and a result of Eliashberg [5] and independently Etnyre [7, if $K \subset S^{3}$ is a knot of genus $g$, then its zero-surgery $S_{0}^{3}(K)$ separates a symplectic manifold. Non-vanishing theorems for the Heegaard Floer invariant $\Phi$ for symplectic four-manifolds, which in turn are built on the symplectic Lefshetz pencils of Donaldson [3, then give a non-vanishing result for the Heegaard Floer $S_{0}^{3}(K)$ from which Equation (6) follows.

These results can be further generalized to give Equation (17). Specifically, an $n$ component link in $S^{3}$ naturally gives rise to a connected knot in the $(n-1)$-fold connected sum of $S^{2} \times S^{1}$. A genus bound analogous to Equation (6) has been shown by $\mathrm{Ni}$ in [25, which in effect establishes Equation (77), in the case where $h$ is one of the $2^{\ell}$ cohomology classes whose evaluation on each meridian for $L$ has absolute value equal to one. Equation (77) then follows from the manner in which the Thurston norm and link Floer homology transform under cabling, see also [16].

As an illustration of Equation (6), consider the Conway link from Figure1. According to the calculations displayed in Figure 6, together with this equation, we conclude that the dual Thurston polytope of the Conway link is as illustrated in Figure 7

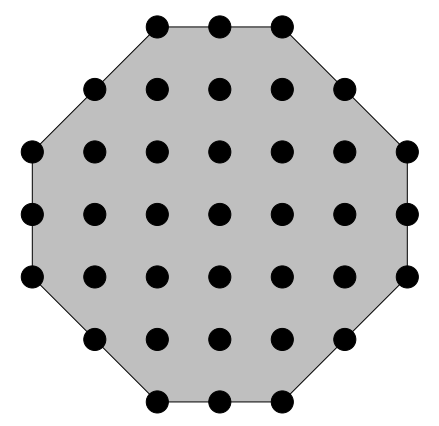

Figure 7. Dual Thurston polytope for the Conway link. Lattice points in $H_{1}\left(S^{3}-L ; \mathbb{Z}\right)$ are indicated by solid circles.

Figure 7 suggests that there are surfaces $F_{1}$ the complement of the Conway link $L$, with $\partial F_{1}$ consisting of a longitude belonging to a first component of $L$, and some number 
of copies of the meridian of the second component, and also with $\chi_{-}\left(F_{1}\right)=5$. In fact, such a surface can be easily obtained by puncturing a genus one Seifert surface for one of the trefoil components in two additional points. A similar surface can be found for the other component of $L$.

Note that a verification of the dual Thurston polytope for the Conway link can be easily obtained by more classical methods (cf. [23]); however, the computation given here is fairly easy (and hopefully illustrates the theory).

5.2. Slice genus. A slice surface for a knot $K$ is a smoothly embedded surface-withboundary $F \subset B^{4}$ which meets $S^{3}$ along its boundary, which is $K$. The slice genus of a knot $g_{*}(K)$ is the minimal genus of any slice surface for $K$. Heegaard Floer homology can be used to give information about this quantity, as follows.

Recall that knot Floer homology is the homology of an associated graded object which is induced by the filtration of a chain complex which calculates $\widehat{H F}\left(S^{3}\right) \cong \mathbb{Z}$. But the entire filtered chain homotopy type of the complex is a knot invariant. Denote the sequence of subcomplexes $F_{i} \subset F_{i+1}$, so that for all sufficiently small integers $i$, $F_{i}=0$, while for all sufficiently large integers, $F_{i}=\widehat{C F}\left(S^{3}\right)$. There is another natural invariant which can be associated to a knot, which is the minimal $i$ for which the map $H_{*}\left(F_{i}\right) \longrightarrow \widehat{H F}\left(S^{3}\right)$ is non-trivial. According to [29] and independently 38,

$$
|\tau(K)| \leq g^{*}(K)
$$

Intriguingly, Rasmussen [39] has shown that a very similar algebraic construction on Khovanov's homology [17, [21, can be used to define a similar (but entirely combinatorial) numerical invariant $s(K)$. Although both $\tau(K)$ and $s(K)$ share many formal properties (and hence agree on many knots), Hedden and Ording have recently shown [15] that these two invariants are in fact distinct. Their examples are certain twisted Whitehead doubles of the trefoil.

5.3. Unknotting numbers. The unknotting number $u(K)$ is the minimal number of crossing changes required to transform $K$ into an unknot. An $n$-step unknotting of a knot $K$ in effect gives an immersed disk in $B^{4}$ with $n$ double-points. Resolving these double-points, we obtain a slice surface for $K$ with genus $n$. This observation immediately verifies the inequality

$$
g^{*}(K) \leq u(K)
$$

For some classes of knots, these two quantities are equal. For example, for the $(p, q)$ torus knot, $g^{*}(K)=u(K)=(p-1)(q-1) / 2$. This was first shown by Kronheimer and Mrowka in [18] (though it has alternative proofs now using either $\tau$ [29] or the Khovanov-Rasmussen invariant $s$ [39]).

But there are Floer-theoretic bounds on $u(K)$ which go beyond the slice genus, cf. [35], [26]. 
Suppose that $K$ has $u(K)=1$. Then, Montesinos observed 24] that the branched double-cover of $S^{3}$ with branching locus $K$, denoted $\Sigma(K)$, can be realized as $\pm d / 2$ surgery on a different knot $C \subset S^{3}$, where here $d=\left|\Delta_{K}(-1)\right|$. Obstructions to this can sometimes be given using Heegaard Floer homology.

To do this in a useful manner, we must understand first $\widehat{H F}(\Sigma(K))$. For some knots, this is a straightforward matter. For example, when $K$ is a knot which admits an alternating projection, an easy induction using Theorem 4.1 shows that $\widehat{H F}(\Sigma(K))$ is a free $\mathbb{Z}$-module of rank $\left|\Delta_{K}(-1)\right|$. This means that the Heegaard Floer homology groups of these three-manifolds is as simple as possible. For any rational homology three-sphere $Y$ (i.e. closed three-manifold with $H_{1}(Y ; \mathbb{Q})=0$ ), the Euler characteristic of $\widehat{H F}(Y)$ is $\left|H_{1}(Y ; \mathbb{Z})\right|$, the number of elements in $H_{1}(Y ; \mathbb{Z})$. A rational homology three-sphere whose homology group $\widehat{H F}(Y)$ is a free module of rank $\left|H_{1}(Y ; \mathbb{Z})\right|$ is called an $L$-space. Thus, if $K$ is a knot with alternating projection, then $\Sigma(K)$ is an $L$-space.

There are obstructions to realizing an $L$-space as surgery on a knot in $S^{3}$, These obstructions are phrased in terms of an additional $\mathbb{Q}$-grading on the Heegaard Floer homology [35, analogous to an invariant defined by Frøyshov [10] in the context of Seiberg-Witten theory. Moreover, this $\mathbb{Q}$-grading can be explicitly calculated for $\Sigma(K)$ for an alternating knot $K$ from its Goeritz matrix. Rather than stating these results precisely, we content ourself here with including a picture of an eight-crossing alternating knot $\left(8_{10}\right.$, see Figure 8) whose unknotting number can be shown to equal two via these (and presently, no other known) techniques.

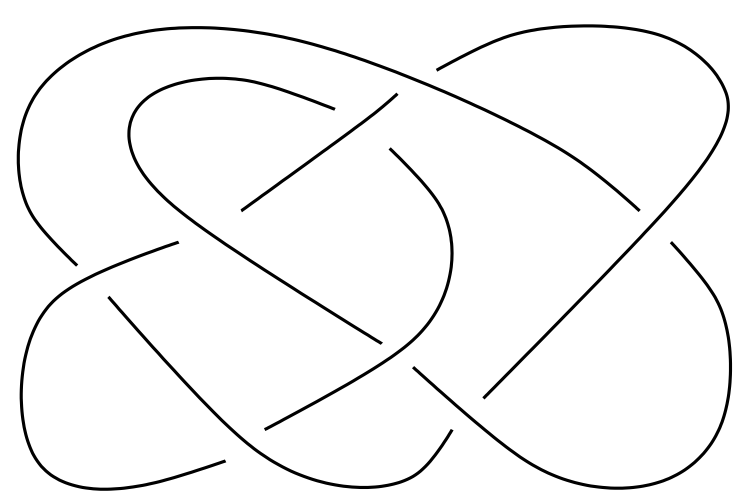

Figure 8. A knot with $u=2$.

Combining these obstructions with recent work of Gordon and Luecke [13], one can classify all knots with 10 and fewer crossings which have unknotting number equal to one. Indeed, a different application of Heegaard Floer homology along similar lines discovered by Owens 26] can be used to complete the unknotting number table for prime knots with nine or fewer crossings. 


\section{REFERENCES}

[1] D. Bar-Natan. Thistlethwaite's link table. http://katlas.math.toronto.edu/wiki/.

[2] P. Braam and S. K. Donaldson. Floer's work on instanton homology, knots, and surgery. In H. Hofer, C. H. Taubes, A. Weinstein, and E. Zehnder, editors, The Floer Memorial Volume, number 133 in Progress in Mathematics, pages 195-256. Birkhäuser, 1995.

[3] S. K. Donaldson. An application of gauge theory to four-dimensional topology. J. Differential Geom., 18(2):279-315, 1983.

[4] S. K. Donaldson. Floer homology groups in Yang-Mills theory, volume 147 of Cambridge Tracts in Mathematics. Cambridge University Press, Cambridge, 2002. With the assistance of M. Furuta and D. Kotschick.

[5] Y. M. Eliashberg. Few remarks about symplectic filling. Geom. Topol., 8:277-293, 2004.

[6] Y. M. Eliashberg and W. P. Thurston. Confoliations. Number 13 in University Lecture Series. American Mathematical Society, 1998.

[7] J. B. Etnyre. On symplectic fillings. Algebr. Geom. Topol., 4:73-80, 2004.

[8] A. Floer. Morse theory for Lagrangian intersections. J. Differential Geometry, 28:513-547, 1988.

[9] A. Floer. Instanton homology and Dehn surgery. In H. Hofer, C. H. Taubes, A. Weinstein, and E. Zehnder, editors, The Floer Memorial Volume, number 133 in Progress in Mathematics, pages 77-97. Birkhäuser, 1995.

[10] K. A. Frøyshov. The Seiberg-Witten equations and four-manifolds with boundary. Math. Res. Lett, 3:373-390, 1996.

[11] D. Gabai. Foliations and the topology of 3-manifolds. J. Differential Geom., 18(3):445-503, 1983.

[12] D. Gabai. Foliations and the topology of 3-manifolds III. J. Differential Geom., 26(3):479-536, 1987.

[13] C. McA. Gordon and J. Luecke. Knots with unknotting number 1 and essential Conway spheres. math.GT/0601265.

[14] M. Gromov. Pseudo holomorphic curves in symplectic manifolds. Inventiones Mathematicae, 82:307-347, 1985.

[15] M. Hedden. The Ozsváth-Szabó and Rasmussen concordance invariants are not equal. math.GT/0512348.

[16] M. Hedden. On knot Floer homology and cabling. Algebr. Geom. Topol., 5:1197-1222 (electronic), 2005.

[17] M. Khovanov. A categorification of the Jones polynomial. Duke Math. J., 101(3):359-426, 2000.

[18] P. B. Kronheimer and T. S. Mrowka. Gauge theory for embedded surfaces. I. Topology, 32(4):773826, 1993.

[19] P. B. Kronheimer and T. S. Mrowka. Scalar curvature and the Thurston norm. Math. Res. Lett., 4(6):931-937, 1997.

[20] P. B. Kronheimer, T. S. Mrowka, P. S. Ozsváth, and Z. Szabó. Monopoles and lens space surgeries. math.GT/0310164.

[21] E-S. Lee. An endomorphism of the Khovanov invariant. Adv. Math., 197(2):554-586, 2005.

[22] P. Lisca and A. I. Stipsicz. Ozsváth-Szabó invariants and tight contact three-manifolds. I. Geom. Topol., 8:925-945, 2004.

[23] C. T. McMullen. The Alexander polynomial of a 3-manifold and the Thurston norm on cohomology. Ann. Sci. de l'Ecole Norm. Sup., 35(2):153-171, 2002.

[24] J. M. Montesinos. Surgery on links and double branched covers of $S^{3}$. In Knots, groups, and 3manifolds (Papers dedicated to the memory of R. H. Fox), pages 227-259. Ann. of Math. Studies, No. 84. Princeton Univ. Press, Princeton, N.J., 1975.

[25] Y. Ni. A note on knot Floer homology of links. math.GT/0506208. 
[26] B. Owens. Unknotting information from Heegaard Floer homology. math.GT/0506485.

[27] P. S. Ozsváth and Z. Szabó. Holomorphic triangles and invariants for smooth four-manifolds. math.SG/0110169.

[28] P. S. Ozsváth and Z. Szabó. Knot Floer homology and rational surgeries. math.GT/0504404.

[29] P. S. Ozsváth and Z. Szabó. Knot Floer homology and the four-ball genus. Geometry and Topology, 7:615-643, 2003.

[30] P. S. Ozsváth and Z. Szabó. Holomorphic disks and genus bounds. Geom. Topol., 8:311-334, 2004.

[31] P. S. Ozsváth and Z. Szabó. Holomorphic disks and knot invariants. Adv. Math., 186(1):58-116, 2004.

[32] P. S. Ozsváth and Z. Szabó. Holomorphic disks and three-manifold invariants: properties and applications. Ann. of Math. (2), 159(3):1159-1245, 2004.

[33] P. S. Ozsváth and Z. Szabó. Holomorphic disks and topological invariants for closed threemanifolds. Ann. of Math. (2), 159(3):1027-1158, 2004.

[34] P. S. Ozsváth and Z. Szabó. Heegaard Floer homology and contact structures. Duke Math. J., 129(1):39-61, 2005.

[35] P. S. Ozsváth and Z. Szabó. Knots with unknotting number one and Heegaard Floer homology. Topology, 44(4):705-745, 2005.

[36] P. S. Ozsváth and Z. Szabó. On knot Floer homology and lens space surgeries. Topology, 44(6):1281-1300, 2005.

[37] J. Rasmussen. Lens space surgeries and a conjecture of Goda and Teragaito. Geom. Topol., 8:10131031, 2004.

[38] J. A. Rasmussen. Floer homology and knot complements. PhD thesis, Harvard University, 2003. math.GT/0306378.

[39] J. A. Rasmussen. Khovanov homology and the slice genus. math.GT/0402131, 2004.

[40] P. Seidel. A long exact sequence for symplectic Floer cohomology. Topology, 42(5):1003-1063, 2003.

[41] W. P. Thurston. A norm for the homology of 3-manifolds, volume 59 of Mem. Amer. Math. Soc., pages $\mathrm{i}-\mathrm{vi}$ and $99-130.1986$.

Department of Mathematics, Columbia University, New York, NY 10027

petero@math. columbia.edu

Department of Mathematics, Princeton University, New Jersey 08544

szabo@math.princeton.edu 